Meta

Journal des tradlucteurs

Translators' Journal

\title{
Une expérience : l'idée d'université...
}

\section{Edmond Robillard}

Volume 15, numéro 1, mars 1970

La traduction religieuse

URI : https://id.erudit.org/iderudit/003963ar

DOI : https://doi.org/10.7202/003963ar

Aller au sommaire du numéro

Éditeur(s)

Les Presses de l'Université de Montréal

ISSN

0026-0452 (imprimé)

1492-1421 (numérique)

Découvrir la revue

Citer cet article

Robillard, E. (1970). Une expérience : l'idée d'université... Meta, 15(1), 26-29.

https://doi.org/10.7202/003963ar d'utilisation que vous pouvez consulter en ligne.

https://apropos.erudit.org/fr/usagers/politique-dutilisation/ 


\section{Une expérience: l'ịdée d’université...}

Au moment où Jeanne d'Arc allait quitter Vaucouleurs, en lui présentant la monture qu'on avait préparée pour elle, on lui demanda : «Savez-vous monter à cheval ? »- «Pas encore, répondit-elle, mais une fois rendue à Orléans, je saurai bien... $\gg$ J'aurais dû faire à peu près la même réponse, en 1957 ou 1958, au moment où je m'imposais comme devoir de vacances de traduire The Idea of a University de J. H. Newman, si on m'avait demandé : "Connaissez-vous l'anglais, ou du moins avez-vous fait une étude exhaustive des œuvres de Newman? » Il n'y a qu'une chose dont je sois assuré, c'est que je les connais beaucoup mieux maintenant.

$\mathrm{Au}$ départ, l'intention était la présentation rapide d'un texte acceptable. La réforme de notre système d'enseignement était commencée, et je croyais faire un travail utile en jetant dans la nacelle de tant de ballons d'essai qu'on lançait alors le lest de réflexions, discutables sans doute, mais en tout cas fondées et élaborées dans un temps et dans un lieu assez éloignés des nôtres pour qu'on ne les crût pas intéressées ou tendancieuses. La traduction, conçue dans cette perspective, était prête à paraître en 1960. Heureusement ou malheureusement, il fut impossible alors de trouver soit un éditeur, soit les fonds nécessaires à l'impression à compte personnel. Le manuscrit était encombrant. Il était difficile de résister à la tentation de relire et de corriger. Je m'engageai dans des recherches biographiques et bibliographiques. Une subvention d'Ottawa mit fin à ce travail de perfectionnement, en me mettant sur le chemin des éditions du Cercle du Livre de France (Canada) et de la collection des textes newmaniens (Desclée de Brouwer, Paris).

Je ne rapporte pas ces faits pour attirer l'attention sur des détails étrangers à l'ouvrage, mais pour qu'on ne s'imagine pas que j'ai l'intention de faire ici figure d'expert. Je n'ai jamais suivi de cours de traduction : on s'en rendra compte à l'imprécision et incorrection de mon vocabulaire, ainsi qu'à la banalité et naïveté des remarques et observations ici rassemblées. J'ai été formé à l'école de la nécessité : obligation, en tant que professeur de théologie, de traduire 
quotidiennement des textes grecs, latins, hébreux, etc., pour le bénéfice d'étudiants qui ne se seraient autrement jamais donné la peine d'aller déchiffrer l'original...

1) La période newmanienne - Newman écrit en longues périodes cicéroniennes, à la manière romantique - bien que ses textes soient chargés d'une érudition et d'une profondeur théologique et philosophique qui dépassent infiniment ce à quoi le romantisme français nous a habitués. J'ai lu, au Caire, le manuscrit d'une traduction de quelques sermons de Newman, calquée sur l'original, et qui rendait vraiment la fluidité et l'incantation du style de l'auteur. J'ai regretté alors de n'avoir pas adopté cette manière; aujourd'hui cependant, à la réflexion, je suis heureux de m'être rendu au conseil que m'avait donné un autre dominicain français : «Coupez ces périodes; présentez le tout en phrases courtes, incisives ».

C'est qu'en effet les Discours universitaires sont beaucoup plus denses que les sermons (je ne parle pas des Sermons universitaires, qui sont presque des thèses de doctorat). Je ne vois pas bien quel lecteur pourrait aujourd'hui saisir du premier coup, sans retours en arrière, le contenu de pensée extrêmement riche et condensé de chaque période newmanienne. Que retenait de ce déluge d'érudition et d'allusions subtiles l'auditeur des Discours de Dublin? Je me le demande. Il attendait probablement, comme nous ferions, la publication de l'œuvre pour rattrapper tout ce qui lui avait échappé !

J'ai donc cru qu'il fallait aider le lecteur pressé. Lui présenter à la petite cuiller ces énormes briques... de substance nutritive. Il ne s'agissait pas pour autant de réduire la qualité de la pensée, ni même de rien ajouter au texte. Comme on ferait aujourd'hui si on reconstruisait Notre-Dame de Paris : remplacer par des colonnes cachées dans les murs les arcs-boutants et contreforts, telle était la conversion à opérer. Sur le plan stylistique, cela veut dire : remplacer par le jeu de la ponctuation et de l'accent verbal les articulations logiques par trop visibles (parce que, étant donné que, etc.). En matière de ponctuation, je n'étais pas plus satisfait de mon travail en corrigeant la dernière épreuve que je ne l'étais en relisant mes premières ébauches; pour moi, c'est le rouet ou le chevalet que le choix d'un point ou d'une virgule! Pour ce qui est du jeu de l'accent verbal, par ailleurs, je le définirais comme suit : l'art de disposer les mots importants (verbes, substantifs, adjectifs) de phrase en phrase sur les positions clés, de telle sorte que l'esprit traverse le paragraphe entier, comme on saute un torrent de pierre en pierre, sans s'y noyer.

Je donne un exemple qui ne paraîtra certainement pas convaincant, mais qui rendra quand même sensible la méthode ici employée. Les intéressés se référeront au texte anglais que je ne donne pas pour ménager l'espace : « Il n'y a pas deux humains, intéressés à vivre heureux ensemble, qui ne devront, si intimes soient-ils, si proches par le goût et la manière de voir, si désireux de ne faire qu'un cœur et qu'une âme, renoncer par égard l'un pour l'autre à bien des préférences et aspirations personnelles... $\gg($ p. 92, §7).

Tout d'abord, il n'y a pas un mot de plus que dans Newman. Les charnières logiques sont toutefois dissimulées. Les propositions se suivent, toutes affirmatives et complètes chacune en elle-même, sans cesser pour autant d'être connexes et interdépendantes. Certes, à lire seulement cette phrase, le lecteur reste sur son appétit, mais il a glané au passage et sans même s'en rendre compte tous les 
éléments qui le conduisent à la conclusion logique, inéluctable, que Newman veut tirer : la vie en société est impossible sans le «compromis». Je dois me tromper, mais il me semble que cette manière rejoint d'aussi près que possible la logique anglaise, en tout cas la logique newmanienne (voir l'Introduction, p. 21) : convaincre par l'apport de faits et de données concrètes, plutôt que par un jeu de distinctions verbales et de nuances conceptuelles. Voilà pourquoi, à mon gré, dans la phraséologie anglaise les jointures sont d'ordinaire si lâches : now et then suffisant à marquer la continuité du développement.

2) Le vocabulaire newmanien - Je ne parlerai pas ici de la difficulté éprouvée : 1) à comprendre le sens que Newman donne à certains termes, au-delà de leur signification courante; 2) à trouver le vocable français qui véhicule le même contenu de pensée et, le cas échéant, la même charge émotive. Ces deux difficultés sont le pain quotidien du traducteur. Je présenterai plutôt deux observations dont les spécialistes feront probablement leurs délices, amusés de ma naïveté et de la candeur avec laquelle je découvrirai devant eux l'Amérique.

D'après moi, tout d'abord, l'anglais est au français ce que le film animé est à la projection de diapositives; autrement dit, en anglais, le mot a toujours un caractère dynamique : il présente la réalité en action, en mouvement; en français, par contre, le mot a un caractère statique, il nous présente la chose " en soi», dans l'immobilité et l'espèce d'éternité que lui confère le processus d'abstraction. En second lieu, mais dans la même ligne de pensée, l'anglais ignore absolument l'abstraction dont se nourrit l'esprit latin; il ne dépasse jamais, en dépit des apparences souvent trompeuses (utilisation de mots d'origine française ou latine, qui pour nous ont une signification abstraite), le niveau de la généralisation pure et simple. Ainsi l'« idée » d'université est, pour un Français, le concept qui exprime l'«essence» de l'université idéale; pour un Anglais, l'«idea» of a University est la somme de tout ce qui vient à l'esprit d'un homme qui a vécu en milieu universitaire et qui sait ce qui se fait et se dit en pareil milieu. Autrement dit encore : l'Anglais ne décolle jamais de l'expérience concrète, tandis que l'esprit latin ne rêve que de passer au royaume des universaux (ce qui explique peut-être pourquoi les Anglais ont souvent emporté le «morceau», pendant que les Français discutaient sur des «mots»: liberté, fraternité, etc.).

En conséquence, pour ne pas franciser indûment Newman, pour respecter sa manière, il fallait éviter le plus possible de donner à ses réflexions et considérations une tournure abstraite. J'ai consacré à ce travail beaucoup d'efforts, et quand les artifices du style n'y ont pas suffi, j'ai prévenu le lecteur en note. Je donne deux exemples de cette précaution dans le choix des vocables concrets : Newman parle d'une certaine perfection que l'éducation libérale donne à l'intelligence. J'ai tout naturellement commencé par traduire : "une perfection de l'intelligence », quitte à m'apercevoir en cours de route que le mot ne «rendait» pas. Pour nous, Latins, une perfection est une espèce d'entité subsistante qui, pour un peu, descendrait dans la rue au premier vent révolutionnaire. Pour Newman une perfection, en ce sens-là, est une «irréalité ». Ce qui existe, pour lui, c'est une intelligence déjà bien «en forme» ou en train de se mettre en forme par une méthode d'entraînement appropriée. Il fallait donc trađuire perfection 
par «perfectionnement» (au sens verbal et dynamique du terme), et non par «perfection » (substantif abstrait et statique).

Un dernier exemple (parmi des centaines). J'ai mis beaucoup de temps à saisir ce que Newman voulait au juste désigner par cette illumination of the mind qui était, selon lui, la fin première visée par l'éducation libérale. J'ai fini par deviner que l'expression n'était pas sans rapport, dans sa pensée, avec le fameux «progrès des lumières» qui avait hanté les nuits des philosophes allemands et français du dix-huitième siècle. Restait à traduire! Je crois avoir trouvé la solution, mais longtemps après la correction des dernières épreuves. Il fallait écrire que l'éducation libérale visait à former des esprits «éclairés》- exactement comme on disait en France à l'époque et dans le même sens philosophique. Encore une fois donc, c'était un adjectif verbal, dynamique, qui rendait exactement l'idée, et non un substantif abstrait et statique, comme «illumination » ou «éclaircissement » ou tout mot de même nature visant à désigner une qualité ou perfection « en soi ».

Je termine ici, ayant couvert l'espace qui m'était généreusement accordé. En curé que je suis, je donnerai une conclusion moralisante à ces considérations. Traduire aide à comprendre ce que pour un chrétien «charité » veut dire. À chaque instant le traducteur doit résister à la tentation de violenter l'autre. Il lui faut prendre le temps de l'apprivoiser et de s'apprivoiser à lui. Il faut déposer tout orgueil et tout désir de l'annexer, de le juger, de le corriger. Il faut plutôt l'écouter et s'ingénier à inventer mille moyens de parvenir jusqu'à lui à travers les espaces infinis de la pensée et du sentiment. Pour moi, traduire Newman a été infiniment plus profitable que n'aurait été un voyage sur la lune : j'ai fait mes premiers pas dans l'âme géniale et fraternelle d'un autre homme. En réalité, le monde ne vit que de traductions : nos conversations ne sont pas autre chose que des traductions simultanées. Malheureusement il y manque souvent l'amour qui aiderait à rendre ces échanges corrects et scientifiquement objectifs.

EDMOND ROBILLARD, O.P. 